\title{
Erratum: Temporal dissipative solitons in a three-level atomic medium confined in a photonic-band-gap fiber [Phys. Rev. A 91, 013828 (2015)]
}

M. Facão, S. Rodrigues, and M. I. Carvalho

(Received 14 October 2015; published 30 October 2015)

DOI: 10.1103/PhysRevA.92.049902

PACS number(s): 42.81.Dp, 42.50.Gy, 42.65.Hw, 99.10.Cd

The parameter $G$ should be corrected to

$$
\begin{aligned}
G= & \frac{c_{1}+c_{2} \Omega_{c}^{2}+c_{3} \Omega_{c}^{4}}{\gamma_{23} \gamma_{33}\left[4\left(\Delta+i \gamma_{12}\right)\left(\delta_{p}-i \gamma_{13}\right)+\Omega_{c}^{2}\right]\left[4\left(\Delta-i \gamma_{12}\right)\left(\delta_{p}+i \gamma_{13}\right) \Omega_{c}+\Omega_{c}^{3}\right]^{2}}, \\
c_{1}= & 32\left(\Delta-i \gamma_{12}\right)^{2}\left(\Delta+i \gamma_{12}\right) \gamma_{13}\left(\delta_{c}^{2}+\gamma_{23}^{2}\right) \gamma_{33}, \\
c_{2}= & -8 i\left(i \Delta+\gamma_{12}\right)\left[12\left(\Delta^{2}+\gamma_{12}^{2}\right) \gamma_{13} \gamma_{23}+\left\{\delta_{c}\left[\gamma_{12}\left(\delta_{p}+\delta_{c}-i \gamma_{13}\right)-2 \Delta \gamma_{13}\right]\right.\right. \\
& \left.\left.+\left[\Delta\left(\delta_{p}-i \gamma_{13}\right)+2 \gamma_{12} \gamma_{13}\right] \gamma_{23}+\gamma_{12} \gamma_{23}^{2}\right\} \gamma_{33}\right], \\
c_{3}= & 2\left\{12 \Delta \gamma_{12} \gamma_{23}+\Delta\left(\gamma_{13}+\gamma_{23}\right) \gamma_{33}+\gamma_{12}\left[-\left(\delta_{p}+\delta_{c}\right) \gamma_{33}-2 i \gamma_{23}\left(6 \gamma_{12}+\gamma_{33}\right)\right]\right\} .
\end{aligned}
$$

This correction only affects the nonlinear parameters $\gamma_{r}$ and $\gamma_{i}$ in a way that affects the graphs of Fig. 6, whose corrected forms are shown here.

If we consider fiber 2 and case 1 , there are $\Delta$ 's for which the equation parameters allow stable solitons. As an example, we consider $\Delta=15 \times 10^{9} \mathrm{~s}^{-1}$ and $\Omega_{c}^{p}=7 \times 10^{11} \mathrm{~s}^{-1}$ which gives $s=1, D=1, \sigma=0.004$, and $v=0.16$. If we further consider $\tau_{0}=50 \mathrm{ps}$, we get $\alpha=0.16$ and $\xi=-0.07$. For this set of parameters, the pulse solution derived above has an amplitude $A=1.3$ and chirp parameter $d=9.5$. In this case, the background instability was only significant around $z=2.6 \mathrm{~m}$. Note that this dissipative soliton should have a peak power of $6.5 \mathrm{~mW}$ since $\gamma_{r}=15 \times 10^{3} \mathrm{~W}^{-1} \mathrm{~m}^{-1}$ and will propagate at $1.9 \%$ of the vacuum light velocity.

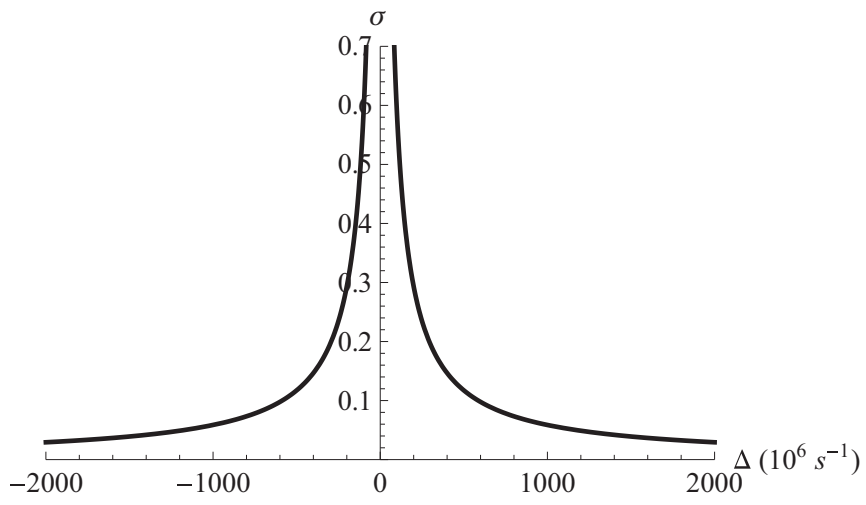

(a)

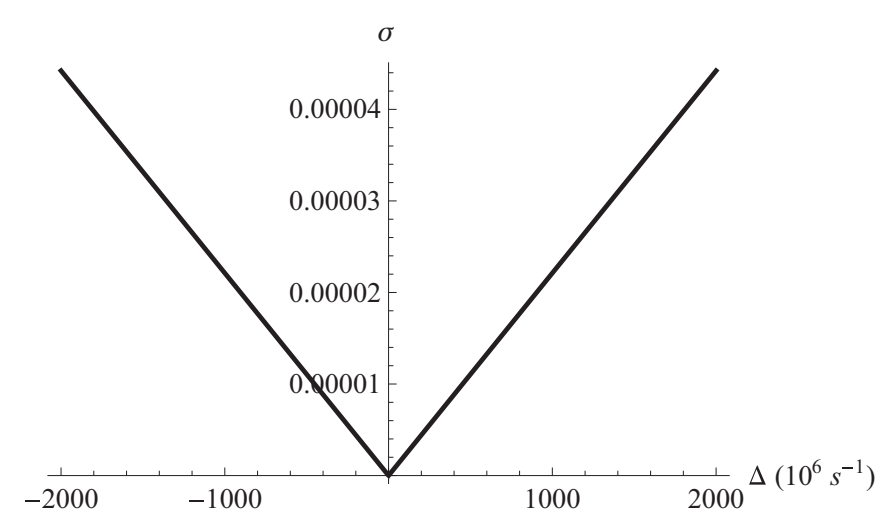

(b)

FIG. 6. Dependence of $\sigma$ with $\Delta$ as calculated using $\sigma=-\operatorname{Im}\left[G\left(\Omega_{c}^{\text {eff }}\right)\right] /\left|\operatorname{Re}\left[G\left(\Omega_{c}^{\text {eff }}\right)\right]\right|$, for (a) case 1 using $\Omega_{c}^{\text {eff }}=7 \times 10^{11} \mathrm{~s}^{-1}$ and (b) case 2 using $\Omega_{c}^{\text {eff }}=7 \times 10^{10} \mathrm{~s}^{-1}$. 